\title{
Modelo de colaboração personalizado em MOOCs
}

\author{
Ana Carla A. Holanda ${ }^{1}$, Patrícia C. A. Restelli Tedesco ${ }^{1}$ \\ ${ }^{1}$ Universidade Federal de Pernambuco (UFPE) \\ Caixa Postal 50.540-740 - Recife - PE - Brasil \\ \{acah@cin.ufpe.br, pcartecin.ufpe.br\}
}

\begin{abstract}
Distance Education (EaD) have evolved bringing the student the conditions to exercise his autonomy of learning. In this scenario, Massive Open Online Courses (MOOCs) are created that are educational environments made available through the Web and aim to make available to a large number of students access to new knowledge. By dealing with a large number of students, MOOCs must constantly encourage the participation and collaboration of those involved. Thus, the research proposal is to investigate how to promote a customized model of collaboration in MOOCs that enables greater engagement and interaction.
\end{abstract}

Keywords. MOOC, Collaboration, Custom Systems

Resumo. A Educação a Distância (EaD) têm evoluído trazendo ao aluno condições para que exercite a sua autonomia de aprendizagem. Neste cenário, surgem os Cursos Abertos Massivos Online (MOOCs) que são ambientes educacionais disponibilizados por meio da Web e objetivam disponibilizar a um grande número de alunos o acesso a novos conhecimentos. Por lidar com um grande número de estudantes, os MOOCs devem estimular constantemente a participação e a colaboração dos envolvidos. Assim, a proposta da pesquisa é investigar como promover um modelo personalizado de colaboração em MOOCs que possibilite um maior engajamento e interação.

Palavras-chave. MOOC, Colaboração, Sistemas Personalizados

\section{Introdução}

MOOC é um modelo baseado na teoria conectivista, na qual não há limites de participantes, restrições de participação ou pré-requisitos, e que utiliza Recursos Educacionais Abertos (REA) (INUZUKA e DUARTE, 2013). Os MOOC se distinguem dos AVA pela quantidade de alunos que podem participar dos cursos. A massividade é um dos principais diferenciais dos cursos MOOC. Segundo Marques (2013), "massivo" define-se por ter mais registrantes do que assistentes e professores capazes de interagir e se comunicar.

Entretanto, muitos são os questionamentos quanto à efetividade dos cursos MOOCs. As pesquisas demonstram que muitos são os desafios para disponibilizar cursos que promovam a aprendizagem colaborativa. Assim, este trabalho tem como objetivo investigar como promover um modelo personalizado de colaboração em ambientes MOOCs que possibilite um maior engajamento e interação. 
VI Congresso Brasileiro de Informática na Educação (CBIE 2017)

Anais do XXVIII Simpósio Brasileiro de Informática na Educação (SBIE 2017)

\section{Metodologia}

Esta pesquisa vai utilizar uma abordagem de estudo de caso exploratório e interpretativo, segundo o preconizado por Merriam (2009). Inicialmente foi realizada uma Revisão Sistemática da Literatura para encontrar as lacunas existentes no que se refere à colaboração em MOOCs. A próxima etapa é a proposição do modelo de colaboração, tendo em vista o resultado da RSL e o mapeamento da arquitetura por meio de um levantamento de características em diversos ambientes, criando assim uma arquitetura de referência que promova interação e engajamento.

O passo seguinte é a implementação do modelo e integração em uma plataforma MOOC. A pesquisa vai apresentar uma abordagem através do estudo de caso com cunho etnográfico em uma plataforma MOOC. Para avaliar o modelo proposto serão realizadas observações, aplicação de questionários e análise dos dados gerados pela plataforma para verificar a eficiência do modelo proposto.

\section{Estado Atual do Trabalho}

A primeira etapa da pesquisa foi a realização da Revisão Sistemática da Literatura (RSL), para identificar as lacunas da área e ajudar a gerar hipóteses de pesquisa. Nessa RSL foram coletados 187 artigos nas bases ACM, IEE, Science Direct e Scopus. A partir da RSL tem-se a seguinte pergunta de pesquisa: "De que forma é possível prover cenários de aprendizagem colaborativos e personalizados em MOOC através da recomendação de pessoas baseados nas características e interesses comuns dos alunos?" Para responder a esta pergunta foi elaborado o seguinte modelo de alto nível, conforme visto na Figura 2, abaixo.

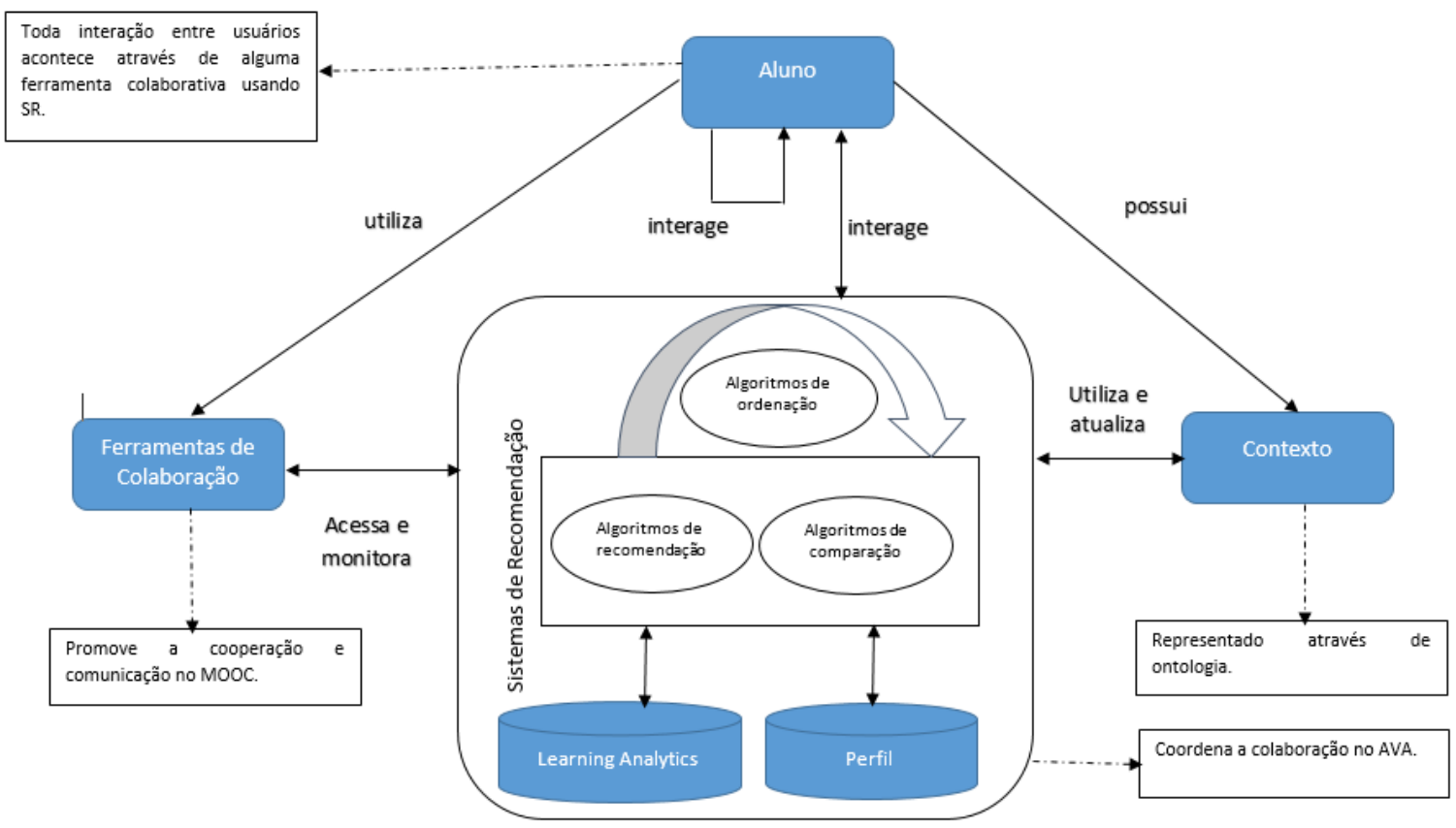

Figura 2: Modelo de colaboração proposto

Será utilizado um Sistema de Recomendação baseado em contexto para recomendar pessoas com o objetivo de formar grupos com interesses comuns e que possam, de alguma forma, promover interação e colaboração. A proposta é utilizar a personalização através da formação de perfis de usuários levando em conta a identidade 
do usuário e a reputação. Análise de Dados de Aprendizagem (Learning Analytics) que é o processo de medição, coleta, análise e divulgação dos dados sobre alunos e seus contextos para fins de compreensão e evolução da aprendizagem e dos ambientes em que ocorre será utilizado. Serão coletados dados baseados nas interações dos alunos e através do método SNA (Social Network Analysis) será interpretada e analisada a estrutura e relações em tarefas colaborativas e interações com as ferramentas de comunicação.

Para realizar essa interpretação serão coletadas informações através do conhecimento do contexto do aluno, pois contexto é o que está por trás da habilidade de definir o que é ou não relevante em um dado momento, e, neste caso em particular permite que o ambiente MOOC filtre e dissemine informações mais úteis e adapte seus serviços às necessidades particulares do estudante, provendo recomendações e, adaptações em interfaces (para que fiquem mais flexíveis e fáceis de usar) (OLIVEIRA, 2013).

Os tipos de interações que ocorrem serão avaliados, além dos períodos e as mídias que são utilizadas para essas participações. O background, a expertise e a experiência dos envolvidos no processo de aprendizagem, além do prestígio ou afinidade de cada um também são características importantes, pois podem determinar a participação de cada um com responsabilidade e colaboração. Além disso, no contexto tecnológico serão verificados os fatores técnicos que podem influenciar a interação social dentro do ambiente online. Um conjunto de perguntas devem ser respondidas assim que os alunos estiverem acesso ao ambiente MOOC. O objetivo é classificá-lo dentre os perfis dos alunos (como visto na pesquisa de OLIVEIRA, 2013). Essa classificação inicial pode ser alterada a partir das interações que existirem.

\section{Considerações Finais}

Como resultados, espera-se que o modelo de colaboração proposto possa realmente garantir cursos MOOC mais colaborativos e que priorizem a busca de uma aprendizagem centrada no interesse individual e coletivo dos alunos aprimorando, desta forma, o seu conhecimento. Atualmente estão sendo planejados os próximos passos referentes a construção do modelo de colaboração e desenvolvimento do sistema de recomendação.

Além disto, a validação do trabalho envolve a realização de um estudo de caso para verificar a eficiência do modelo proposto.

\section{Referências}

Inuzuka, Marcelo Akira. Duarte, Rafael Teixeira. (2012). Produção de REA apoiada por MOOC. In: Santana; Bianca. Rossini, Carolina. Pretto, Nelson de Lucca (Organizadores). Recursos Educacionais Abertos: práticas colaborativas políticas públicas.1. ed., 1 imp. - Salvador: Edufba; São Paulo: Casa da Cultura Digital.

Marques, J. (2013). "What is a Massive Open Online Course Anyway? MN+R Attempts a Definition". Available at: http://moocnewsandreviews.com/what-is-a-massive-openonline-course-anyway-attempting-definition/. Acessado em 14.08.2016.

Merriam, Sharan B. (2009). Qualitative research: a guide to design and implementation.

Oliveira, Eduardo Araújo. i-collaboration 3.0: um framework de apoio ao desenvolvimento de Ambientes Distribuídos de Aprendizagem Sensíveis ao Contexto. Tese Doutorado. Universidade Federal de Pernambuco. Cin - Ciência da computação, 2013. 